\title{
Association between Mitofusin 2 Gene Polymorphisms and Late-Onset Alzheimer's Disease in the Korean Population
}

\author{
Young Jong Kim¹, Jin Kyung Park', Won Sub Kang ${ }^{1}$, Su Kang Kim², Changsu Han ${ }^{3}$, Hae Ri $\mathrm{Na}^{4}$, \\ Hae Jeong Park ${ }^{2}$, Jong Woo Kim', Young Youl Kim ${ }^{5}$, Moon Ho Park ${ }^{6}$, and Jong-Woo Paik ${ }^{\circledR}$ \\ 1'Department of Neuropsychiatry, School of Medicine, Kyung Hee University, Seoul, Republic of Korea \\ ${ }^{2}$ Kohwang Medical Research Institute, School of Medicine, Kyung Hee University, Seoul, Republic of Korea \\ ${ }^{3}$ Psychiatric Department, Korea University Ansan Hospital, Ansan, Republic of Korea \\ ${ }^{4}$ Department of Neurology, Bobath Memorial Hospital, Seongnam, Republic of Korea \\ ${ }^{5}$ Division of Brain Diseases, Center for Biomedical Science, National Institute of Health Osong Health Technology Administration Complex, Cheongju, \\ Republic of Korea \\ ${ }^{6}$ Department of Neurology, Korea University Ansan Hospital, Ansan, Republic of Korea
}

Objective Mitochondrial dysfunction is a prominent and early feature of Alzheimer's disease (AD). The morphologic changes observed in the $\mathrm{AD}$ brain could be caused by a failure of mitochondrial fusion mechanisms. The aim of this study was to investigate whether genetic polymorphisms of two genes involved in mitochondrial fusion mechanisms, optic atrophy 1 (OPA1) and mitofusin 2 (MFN2), were associated with AD in the Korean population by analyzing genotypes and allele frequencies.

Methods One coding single nucleotide polymorphism (SNP) in the MFN2, rs1042837, and two coding SNPs in the OPA1, rs7624750 and rs9851685, were compared between 165 patients with AD (83 men and 82 women, mean age $72.3 \pm 4.41)$ and 186 healthy control subjects (82 men and 104 women, mean age 76.5 \pm 5.98 ).

Results Among these three SNPs, rs1042837 showed statistically significant differences in allele frequency, and genotype frequency in the co-dominant 1 model and in the dominant model.

Conclusion These results suggest that the rs1042837 polymorphism in MFN2 may be involved in the pathogenesis of AD.

Psychiatry Investig 2017;14(1):81-85

Key Words MFN2, Mitochondrial fusion, Alzheimer's disease.

\section{INTRODUCTION}

Many studies indicate that mitochondrial dysfunction is a prominent and early feature of Alzheimer's disease (AD)., ${ }^{1,2}$ Mitochondrial dysfunction occurs early in $\mathrm{AD}$ pathogenesis and precedes the development of plaque formation. Impaired mitochondrial function leads to increased oxidative stress that enhances $A \beta$ production. ${ }^{3}$ Mitochondria are dynamic organelles that can divide and fuse with each other. Damaged mitochondria undergo fusion and recover their function. Impaired

Received: February 11, 2016 Revised: March 21, 2016 Accepted: March 28, 2016 Available online: May 27, 2016

$\triangle$ Correspondence: Jong-Woo Paik, MD, PhD

Department of Neuropsychiatry, School of Medicine, Kyung Hee University, 23 Kyungheedae-ro, Dongdaemun-gu, Seoul 02447, Republic of Korea Tel: +82-2-958-8419, Fax: +82-2-957-1997, E-mail: paikjw@gmail.com

(a) This is an Open Access article distributed under the terms of the Creative Commons Attribution Non-Commercial License (http://creativecommons.org/licenses/bync/3.0) which permits unrestricted non-commercial use, distribution, and reproduction in any medium, provided the original work is properly cited. mitochondrial fusion ability makes cells more sensitive to apoptotic stimuli, therefore mitochondrial fusion is considered to be a protective mechanism. ${ }^{1,4}$ In $\mathrm{AD}$ brains, the mitochondria were smaller, rounder, and had elongated disruptions of the cristae in contrast to normal controls. ${ }^{5}$ These ultrastructural alternations could be caused by a failure of the mitochondrial fusion mechanism. Mitochondrial fusion occurs in both the inner and outer membranes.

Mitochondrial fusion is mediated by three GTPase dynamin-like proteins, mitofusin 1 (MFN1), mitofusin 2 (MFN2), and optic atrophy 1 (OPA1). MFN1 and MFN2 mediate mitochondrial outer membrane fusion, whereas OPA1 is responsible for inner membrane fusion. ${ }^{4}$ Human autopsy studies have reported reduced expression of these fusion proteins in $\mathrm{AD}$ hippocampal tissues. ${ }^{6}$ The protein involved in mitochondrial membrane fusion is an essential component of mitochondrial DNA (mtDNA) homeostasis. Decreased OPA1 or MFN 1/2 causes mtDNA depletion, the perinuclear accumulation of mi- 
tochondria, and oxidative stress. ${ }^{7-9}$ Furthermore, in recent study using N2a cells, Mfn2 overexpression led to the inhibition of $A \beta$ oligomers, which mediated cell death.

Therefore, we hypothesized that the genes involved in mitochondrial fusion could be good candidate genes for involvement in $\mathrm{AD}$ pathogenesis. Additionally, the MFN2 is located on chromosome 1p36, which was suggested as one of $\mathrm{AD}$-associated chromosomal loci. ${ }^{10}$ The aim of this study was to investigate whether genetic polymorphisms of the OPA1, MFN1, and MFN2 were associated with $\mathrm{AD}$ in the Korean population by analyzing their genotype and allele frequencies. Additionally, we analyzed the relationship between genetic polymorphisms of these genes and the severity of the behavioral and psychological symptoms of dementia (BPSD) based on clinical assessment.

\section{METHODS}

\section{Subjects}

The studied sample consisted of $165 \mathrm{AD}$ patients (83 men and 82 women; mean age $72.3 \pm 4.4$ years old; range $65-81$ ) and 186 healthy controls (82 men and 104 women; mean age $76.5 \pm 5.9$ years old; range 67-92). There was no significant difference of gender ratio between two groups $(p=0.253)$, however the mean ages of two groups were different $(\mathrm{p}<0.001)$. Therefore, the statistical data in this study were analyzed following adjustment for age and gender as covariates. All $\mathrm{AD}$ patients and healthy controls were recruited from five university hospitals and two geriatric hospitals which had participated in Dementia database project in Korea. The diagnosis of AD was based on the National Institute of Neurological and Communicative Disorders and Stroke and the Alzheimer's Disease and Related Disorders Association "probable AD" criteria. ${ }^{11}$ All controls were cognitively intact and free of significant illness, following medical history and clinical interview. $\mathrm{AD}$ patients were assessed using the Korean version of the Consortium to Establish a Registry for Alzheimer's Disease-Neuropsychological Assessment Battery (CERAD-NP), ${ }^{12}$ The Neuropsychiatric Inventory (NPI), ${ }^{13}$ and the Short Form Geriatric Depression Scale-Korean Version (S-GDS-K). ${ }^{14}$ Peripheral blood samples from each subject were drawn and $10 \mathrm{~mL}$ of blood was used for DNA extraction. Written informed consent was obtained from all participants involved in the study or their legal guardians. The study was approved by the institutional review board of each participating hospital.

\section{Single nucleotide polymorphism selection and genotyping}

We searched the coding SNPs of the MFN1, MFN2, and OPA1 using the SNP database of the National Center for Bio- technology Information (http://www.ncbi.nlm.nih.gov/SNP). SNPs with heterozygosity below 0.1 , minor allele frequency (MAF) below 0.05, or unknown genotype frequency in Asian populations were excluded. Of the SNPs of the MFN1, all coding SNPs had a MAF below 0.05 or heterozygosity below 0.1 . rs1042837 (Ser523Ser) of the MFN2 and rs7624750 (Ser158Asn) and rs9851685 (Ala703Ala) of the OPA1 fulfilled our criteria. Thus, these three SNPs were selected and genotyped. A map of the positions of the three SNPs under investigation is shown in Figure 1. The genotypes of two OPA1 SNPs were determined by Sequenom MassARRAY technology (Sequenom iPLEX assay, San Diego, CA, USA). Primers were designed using the Assay Designer 3.1 (Sequenom) and DNA samples were amplified by multiplex PCR reactions. Genotyping and data analysis were performed using the Sequenom MassARRAY iPLEX platform and the MassARRAY Typer 4.0, respectively. rs1042837 of the MFN2 was not amenable to genotyping by the Sequenom MassARRAY technology, thus it was directly sequenced. Primers for rs1042837 were sense, 5'-ACT TGA CTG GGG TGT GGT TC-3' and antisense, 5'-TCT GGA GGC AGG GTA CAG AC-3'. DNA sequencing and data analysis were performed using the ABI PRISM 3730XL analyzer (PE Applied Biosystems, Foster City, CA, USA) and SeqManII software (DNAStar Inc., Madison, WI, USA).

\section{Statistical analysis}

Analysis of the genotype frequencies and Hardy-Weinberg equilibrium (HWE) was performed using SNPStats (http:// bioinfo.iconcologia.net/snpstats/start.htm) and SPSS version 18.0 (IBM, Armonk, NY, USA). Multiple logistic regression models were built to analyze genetic data: co-dominant 1 (major allele homozygotes vs. heterozygotes), co-dominant 2 (major allele homozygotes vs. minor allele homozygotes), dominant (major allele homozygotes vs. heterozygotes and minor allele homozygotes), and recessive (major allele homozygotes and heterozygotes vs. minor allele homozygotes). The scores

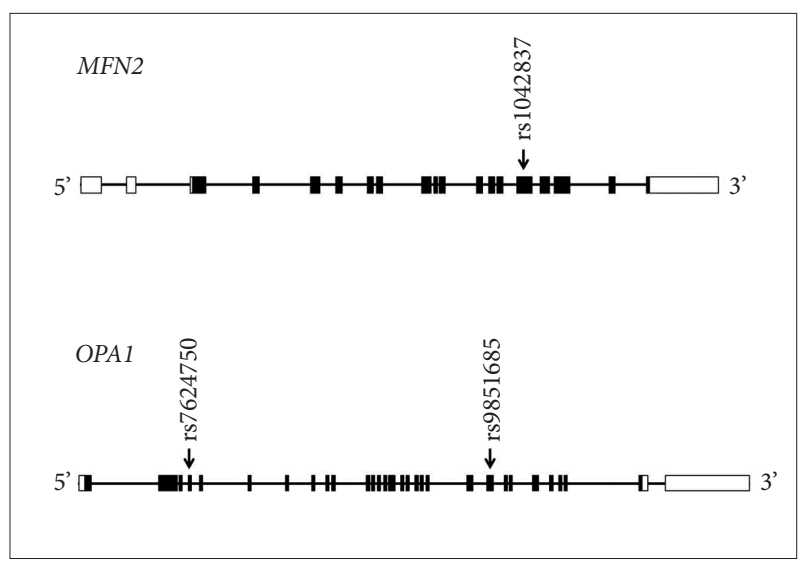

Figure 1. Map of three polymorphisms under investigation. 
(frequency x severity) of twelve NPI items in the AD patients were compared with the genetic polymorphisms using Kruskal-Wallis tests (for the co-dominant model) and Mann-Whitney $U$ tests (for the dominant and recessive model) using SPSS version 18.0. Bonferroni correction was applied by lowering the significance level of $\mathrm{p}$ values to $0.016(=0.05 / 3)$ to avoid chance findings due to multiple testing.

\section{RESULTS}

The genotype distributions of the SNPs in both $\mathrm{AD}$ patients and the controls were in HWE ( $p>0.05$; data not shown). Table 1 shows the genotype and allele frequencies of the three examined SNPs. In the present study, rs1042837 of the MFN2 was associated with the risk of $\mathrm{AD}$, but no significant differences were found for the two SNPs of the OPA1 between AD patients and controls. The genotype frequency of rs1042837 was significantly different between the two groups $[\mathrm{p}=0.015$, odds ratio $(\mathrm{OR})=0.45,95 \%$ confidence interval $(\mathrm{CI})=0.23-0.86$ in the co-dominant model 1 , and $\mathrm{p}=0.0075, \mathrm{OR}=0.43,95 \% \mathrm{CI}=$ $0.23-0.81$ in the dominant model]. The allele frequency of rs1042837 was also significantly different between the AD group and the control group ( $\mathrm{p}=0.0059)$. In the haplotype analysis using Haploview 4.2 program, a linkage disequilibrium block was made between two SNPs of OPA1 ( $\mathrm{D}^{\prime}=1, \mathrm{r}^{2}=0.88$ ), however, haplotype frequencies showed no significant difference between $\mathrm{AD}$ and controls (data not shown). The analysis of the relationship between genotype and BPSD severity as measured by NPI revealed no statistically significant findings (data not shown). Although the symptom of 'disinhibition' was associated with rs1042837 ( $\mathrm{p}=0.033)$ in the dominant model and the symptom of 'irritability' was associated with rs7624750 $(p=0.032)$ in the recessive model, these results did not meet the $\mathrm{p}$ value criteria of this study.

\section{DISCUSSION}

In the present study, we found a significant association be-

Table 1. Genotype and allele frequencies of MFN2 and OPA1 SNPs in control subjects and AD patients

\begin{tabular}{|c|c|c|c|c|c|c|c|c|}
\hline \multirow{2}{*}{ SNP } & \multirow{2}{*}{ Type } & \multicolumn{2}{|c|}{ Control } & \multicolumn{2}{|c|}{$\mathrm{AD}$} & \multirow{2}{*}{ Model } & \multirow{2}{*}{ OR (95\% CI) } & \multirow{2}{*}{$\mathrm{p}$-value } \\
\hline & & $\mathrm{N}$ & $\%$ & $\mathrm{~N}$ & $\%$ & & & \\
\hline rs1042837 & Genotype & & & & & & & \\
\hline \multirow[t]{7}{*}{$(M F N 2)$} & $\mathrm{C} / \mathrm{C}$ & 144 & 77.4 & 146 & 88.5 & Co-dominant 1 & $0.45(0.23-0.86)$ & $0.015^{*}$ \\
\hline & $\mathrm{C} / \mathrm{T}$ & 39 & 21.0 & 18 & 10.9 & Co-dominant 2 & $0.27(0.03-2.70)$ & 0.264 \\
\hline & $\mathrm{T} / \mathrm{T}$ & 3 & 1.6 & 1 & 0.6 & Dominant & $0.43(0.23-0.81)$ & $0.0075^{*}$ \\
\hline & & & & & & Recessive & $0.31(0.03-3.07)$ & 0.28 \\
\hline & Allele & & & & & & & \\
\hline & $\mathrm{C}$ & 327 & 87.9 & 310 & 93.9 & & & \\
\hline & $\mathrm{T}$ & 45 & 12.1 & 20 & 6.1 & & & $0.0059^{*}$ \\
\hline rs7624750 & Genotype & & & & & & & \\
\hline \multirow[t]{7}{*}{$(O P A 1)$} & $\mathrm{G} / \mathrm{G}$ & 82 & 44.1 & 73 & 44.2 & Co-dominant 1 & $1.21(0.75-1.97)$ & 0.44 \\
\hline & $\mathrm{G} / \mathrm{A}$ & 75 & 40.3 & 76 & 46.1 & Co-dominant 2 & $0.65(0.31-1.35)$ & 0.249 \\
\hline & $\mathrm{A} / \mathrm{A}$ & 29 & 15.6 & 16 & 9.7 & Dominant & $1.05(0.67-1.66)$ & 0.83 \\
\hline & & & & & & Recessive & $0.59(0.30-1.19)$ & 0.13 \\
\hline & Allele & & & & & & & \\
\hline & G & 239 & 64.2 & 222 & 67.3 & & & \\
\hline & A & 133 & 35.8 & 108 & 32.7 & & & 0.3995 \\
\hline rs9851685 & Genotype & & & & & & & \\
\hline \multirow[t]{7}{*}{$(O P A 1)$} & $\mathrm{T} / \mathrm{T}$ & 88 & 47.3 & 78 & 47.3 & Co-dominant 1 & $1.21(0.75-1.95)$ & 0.446 \\
\hline & $\mathrm{C} / \mathrm{T}$ & 75 & 40.3 & 73 & 44.2 & Co-dominant 2 & $0.75(0.34-1.63)$ & 0.465 \\
\hline & $\mathrm{C} / \mathrm{C}$ & 23 & 12.4 & 14 & 8.5 & Dominant & $1.10(0.70-1.72)$ & 0.69 \\
\hline & & & & & & Recessive & $0.69(0.32-1.45)$ & 0.32 \\
\hline & Allele & & & & & & & \\
\hline & $\mathrm{T}$ & 251 & 67.5 & 229 & 67.0 & & & \\
\hline & $\mathrm{C}$ & 121 & 32.5 & 101 & 33.0 & & & 0.5849 \\
\hline
\end{tabular}

*statistically significant values ( $\mathrm{p}<0.016)$. SNP: single nucleotide polymorphism, AD: Alzheimer's disease, OR: odds ratio, CI: confidence interval 
tween the rs1042837 SNP in the MFN2 and AD risk in Korean patients. Particularly, T allele frequency in the controls was significantly more than that in $\mathrm{AD}$ patients. This finding suggests that the T allele of rs1042837 might be a protective factor for risk of $\mathrm{AD}$.

Mutations in the MFN2 cause an autosomal dominant disease known as Charcot-Marie-Tooth disease subtype 2A (CMT2A), which is characterized by motor and sensory peripheral neuropathy. Although CMT2A mainly affects the peripheral nervous system, recent reports indicate that CMT2A might cause brain pathologies. In one study using brain MRI, 7 of 18 early-onset CMT patients with MFN2 mutations showed brain abnormalities in gray matter and/or white matter. ${ }^{15}$ In another study, three family members who carried MFN2 missense mutations showed signs of cognitive impairment such as delayed language development and low scores on the Wechsler Intelligence Scale. ${ }^{16}$ These reports support the possibility that the MFN2 mutation affects central nervous system as well as peripheral nervous system.

Many findings that suggest mitochondrial dysfunction, such as increased free radical production, activated apoptosis process, defective glucose metabolism, abnormal mitochondrial morphology, and altered mtDNA are observed in $\mathrm{AD} .{ }^{7,17}$ Swerdlow et al, ${ }^{2,18,19}$ proposed the $\mathrm{AD}$ mitochondrial cascade hypothesis. This suggests that as mitochondrial function declines with aging, the failure of neuronal compensation leads to bioenergetics hyper-metabolism and thus the increased production and accumulation of $A \beta$. Tau phosphorylation is also facilitated by mitochondrial dysfunction. Gan et al. ${ }^{20} \mathrm{ex}$ perimented with cybrid cells made of mtDNA-depleted cells fused with the platelet cytoplasm of $\mathrm{AD}$ patients or control subjects. The mitochondria of the AD cybrids were bleb-like in shape, shorter in length than controls, and Mfn2 was significantly decreased in the mitochondrial fraction of the $\mathrm{AD}$ cybrids. In the histologic findings of the six MFN2 mutation cases, small and spherical mitochondria were observed ${ }^{21}$ and these morphologic changes were similar to those observed in $\mathrm{AD}$ brain tissue. ${ }^{5}$ In this respect, the MFN2 might be a promising candidate for further investigation in the pathogenesis of AD.

The present study had some limitations. The major limitation was the small sample size in present study, therefore, some type I errors may have existed. Our findings should be considered only preliminary, requiring further validations in larger samples, and possibly in other ethnic group. Secondly, we analyzed only coding SNPs, which may not fully tag all the genetic variants in the OPA1 and MFN2 genes. An additional analysis of variants in another region in these genes could reveal the role of the OPA1 and MFN2 gene polymorphisms to susceptibility to $\mathrm{AD}$. Despite these limitations, to our knowledge, this is the first report showing that polymorphisms in MFN2 were associated with $\mathrm{AD}$.

In conclusion, our study revealed a significant association between the rs1042837 SNP of the MFN2 and AD in the Korean population. These findings suggest that genetic polymorphisms of mitochondrial fusion genes might be involved in the pathogenesis of $\mathrm{AD}$.

\section{Acknowledgments}

The authors thank the subjects for their willingness to participate in this study. This study was supported by Korea National Institute of Health Intramural Research Grant (2010E6200100) and a research grant from the Kyung Hee University College of Medicine.

\section{REFERENCES}

1. Santos RX, Correia SC, Wang X, Perry G, Smith MA, Moreira PI, et al. A synergistic dysfunction of mitochondrial fission/fusion dynamics and mitophagy in Alzheimer's disease. J Alzheimers Dis 2010;20(Suppl 2):S401-S412.

2. Swerdlow RH, Burns JM, Khan SM. The Alzheimer's disease mitochondrial cascade hypothesis. J Alzheimers Dis 2010; 20(Suppl 2):S265-S279.

3. Leuner K, Schulz K, Schutt T, Pantel J, Prvulovic D, Rhein V, et al. Peripheral mitochondrial dysfunction in Alzheimer's disease: focus on lymphocytes. Mol Neurobiol 2012;46:194-204.

4. Hoppins S, Lackner L, Nunnari J. The machines that divide and fuse mitochondria. Annu Rev Biochem 2007;76:751-780.

5. Baloyannis SJ. Mitochondrial alterations in Alzheimer's disease. J Alzheimers Dis 2006;9:119-126.

6. Wang X, Su B, Lee HG, Li X, Perry G, Smith MA, et al. Impaired balance of mitochondrial fission and fusion in Alzheimer's disease. J Neurosci 2009;29:9090-9103.

7. Zhu X, Perry G, Smith MA, Wang X. Abnormal mitochondrial dynamics in the pathogenesis of Alzheimer's disease. J Alzheimers Dis 2013; 33(Suppl 1):S253-S262.

8. Cho DH, Nakamura T, Lipton SA. Mitochondrial dynamics in cell death and neurodegeneration. Cell Mol Life Sci 2010;67:3435-3447.

9. Ranieri M, Brajkovic S, Riboldi G, Ronchi D, Rizzo F, Bresolin N, et al. Mitochondrial fusion proteins and human diseases. Neurol Res Int 2013; 2013:293893.

10. Hiltunen M, Mannermaa A, Thompson D, Easton D, Pirskanen M, Helisalmi S, et al. Genome-wide linkage disequilibrium mapping of lateonset Alzheimer's disease in Finland. Neurology 2001;57:1663-1668.

11. McKhann G, Drachman D, Folstein M, Katzman R, Price D, Stadlan EM. Clinical diagnosis of Alzheimer's disease: report of the NINCDSADRDA Work Group under the auspices of Department of Health and Human Services Task Force on Alzheimer's Disease. Neurology 1984;34: 939-944.

12. Morris JC, Heyman A, Mohs RC, Hughes JP, van Belle G, Fillenbaum G, et al. The Consortium to Establish a Registry for Alzheimer's Disease (CERAD). Part I. Clinical and neuropsychological assessment of Alzheimer's disease. Neurology 1989;39:1159-1165.

13. Cummings JL, Mega M, Gray K, Rosenberg-Thompson S, Carusi DA, Gornbein J. The Neuropsychiatric Inventory: comprehensive assessment of psychopathology in dementia. Neurology 1994;44:2308-2314.

14. Jung IK, Kwak DI, Joe SH, Lee HS. A study of standarization of Korean form of geriatric depression scale. J Korean Geriatr Psychiatry 1997;1:6172 .

15. Chung KW, Suh BC, Cho SY, Choi SK, Kang SH, Yoo JH, et al. Earlyonset Charcot-Marie-Tooth patients with mitofusin 2 mutations and brain involvement. J Neurol Neurosurg Psychiatry 2010;81:1203-1206.

16. Del Bo R, Moggio M, Rango M, Bonato S, D’Angelo MG, Ghezzi S, et al. Mutated mitofusin 2 presents with intrafamilial variability and brain 
mitochondrial dysfunction. Neurology 2008;71:1959-1966

17. Hroudova J, Singh N, Fisar Z. Mitochondrial dysfunctions in neurodegenerative diseases: relevance to Alzheimer's disease. Biomed Res Int 2014;2014:175062.

18. Swerdlow RH, Burns JM, Khan SM. The Alzheimer's disease mitochondrial cascade hypothesis: progress and perspectives. Biochim Biophys Acta 2014;1842:1219-1231.

19. Swerdlow RH. Brain aging, Alzheimer's disease, and mitochondria. Biochim Biophys Acta 2011;1812:1630-1639.
20. Gan X, Huang S, Wu L, Wang Y, Hu G, Li G, et al. Inhibition of ERKDLP1 signaling and mitochondrial division alleviates mitochondrial dysfunction in Alzheimer's disease cybrid cell. Biochim Biophys Acta 2014;1842:220-231.

21. Vallat JM, Ouvrier RA, Pollard JD, Magdelaine C, Zhu D, Nicholson GA, et al. Histopathological findings in hereditary motor and sensory neuropathy of axonal type with onset in early childhood associated with mitofusin 2 mutations. J Neuropathol Exp Neurol 2008;67:1097-1102. 